\title{
SHORT AND OPEN CIRCUITED SLOT LINE
}

\author{
Jan Macháč, Ján Zehentner \\ Faculty of Electrical Engineering \\ Czech Technical University \\ Technická 2, 16627 Prague, Czech Republic \\ Wolfgang Menzel \\ University of Ulm \\ Albert Einstein Allee 41, 89069 Ulm, Germany
}

\begin{abstract}
A slot line terminated by the short and open end is analysed by the spectral domain method (SDM). The original process of Yang and Alexópoulos is modified by application of the piecewise sinusoidal functions only. Reflection coefficient is obtained by fitting the field along the slot described by a superposition of these functions to the standing wave pattern by the least squares method. Closed form formulae of the normalized impedance of the slot line terminated by the short have arisen from fitting its values computed by the SDM. They facilitate CAD of circuits. Full wave analysis of the open-circuited slot line is performed. Agreement between theory and experiment and between our results and data published in the literature is very good.
\end{abstract}

\section{INTRODUCTION}

Importance of uniplanar structures in MIC is widely known. Their main representatives are slot line, coplanar waveguide, coplanar strips and a series of discontinuities made of slots of various shapes inclusive of slot line stubs. Stubs terminate either by a short or by an open end. Open-circuited slot line termination can be made like rectangular, circular or fan patch etched in the metallization. In fact, both short- and open-end terminations are nonideal. Short-end has non-zero complex impedance and open-end has it finite, again complex. Proper end impedance should be accounted in the precise circuit design.

The slot line short was analysed in [1], [2] and [3]. Yang and Alexópoulos [1] described field in the plane of a slot by piecewise sinusoidal functions (PWSF) together with incident and reflected waves. Reflection coefficient, as an additional quantity, is found then strightforward from a set of linear equations written for amplitudes of the PWSF's. Rozzi et al [2] sampled incident and reflected waves by PWSF's only. Short- and open-circuited slot lines were analysed by FD-TD method in [3]. Data in [3] are presented in the very wide frequency range. However, no evidence is given whether leaky waves are accounted. Measured data concerning short- and open-circuited slot line are in [4] and [5].

Results presented in this paper have arisen by the SDM [1]. The field along the slot is described by PWSF's only. Alternative and original determination of the reflection coefficient is proposed. Computed data are comparable well with those ones given in the literature [1], [2], [5] and have been verified experimentally.

An exact model of the short-circuited slot line based on SDM is not suitable for insertion into CAD packages. For this purpose closed-form formulae have been derived fitting the computed normalized terminal impedance by the least squares method.

Normalized terminal impedance of the open-circuited slot line with rectangular slot-patch calculated by the SDM is presented. Theoretical values of the reflection coefficient have been verified by measurement.

\section{APPROACH TO THE PROBLEM}

Two investigated uniplanar slot structures are in Fig. 1. In a full wave analysis of the short- and open-circuited slot line the well known spectral domain method, described e.g. in [1], was used. Relations between current densities $J_{x}, J_{y}$ and electric fields $E_{x}, E_{y}$ follow from the boundary conditions in the plane of the metallization. After their $2 D$ transformation into the spectral domain

$$
\begin{aligned}
& \tilde{J}_{x}=\tilde{G}_{x x} \tilde{E}_{x}+\tilde{G}_{x y} \tilde{E}_{y} \\
& \tilde{J}_{y}=\tilde{G}_{y x} \tilde{E}_{x}+\tilde{G}_{y y} \tilde{E}_{y}
\end{aligned}
$$

where $\tilde{\mathrm{G}}$ are transforms of proper Green's functions. A couple of integral equations for unknown field distributions $E_{x}(x, y), E_{y}(x, y)$ follows from the requirement set on the current densities $J_{x}, J_{y}$ within the area without the 
metallization

$$
\mathrm{J}_{\mathrm{x}}(\mathrm{x}, \mathrm{y})=0, \quad \mathrm{~J}_{\mathrm{y}}(\mathrm{x}, \mathrm{y})=0
$$

These equations are solved by the Galerkin's method in the spectral domain.

A sum of the PWSF's

$$
f_{x n}(x, y)=\frac{\sin \left[k_{e}(d-|y-n d|)\right]}{\sin \left(k_{e} d\right)} \frac{1}{\sqrt{1-(2 x / w)^{2}}}, \quad|x| \leq w / 2,|y-n d| \leq d
$$

gives the transversal electric field $E_{x}$ in the slot while the longitudinal component $E_{y}$ is neglected there. In (4) $n$ is an integer numbering the functions, $d$ is the half-length of the PWSF and $k_{e}=k_{0} \sqrt{\left(1+\varepsilon_{r}\right) / 2}$ as in [1]. For the open-circuited slot line both $E_{x}$ and $E_{y}$ have to be used within the area of the etched patch, Fig. 1b. They are

$$
\begin{aligned}
f_{x n}(x, y) & =\frac{\sin \left[k_{e}\left(d_{x}-\left|y-i_{n} d_{x}\right|\right)\right]}{\sin \left(k_{e} d_{x}\right)} \\
f_{y m}(x, y) & =\frac{\sin \left[k_{e}\left(d_{y}-\left|x-i_{m} d_{x}\right|\right)\right]}{\sin \left(k_{e} d_{y}\right)}
\end{aligned}
$$

and hold on rectangular cells covering the patch, positions of these cells specify $i_{n}, i_{m}$. As in (4) integers $n$ and $m$ number the PWSF's, $d_{x}$ and $d_{y}$ are respective half-lengths of the PWSF's. However, again PWSF's (4) describe the field in the slot. Final set of equations is

$$
\begin{array}{ll}
\int_{-\infty}^{\infty}\left(\tilde{G}_{x x} \tilde{E}_{x}+\tilde{G}_{x y} \tilde{E}_{y}\right) \tilde{F}_{x n} d_{k} d k_{y}=0, & n=1, \ldots, N \\
\int_{-\infty}^{\infty}\left(\tilde{G}_{y x} \tilde{E}_{x}+\tilde{G}_{y y} \tilde{E}_{y}\right) \tilde{F}_{y m} d_{k} d k_{y}=0, & m=1, \ldots, M
\end{array}
$$

where $k_{x}, k_{y}$ are spectral variables, $\tilde{F}_{x n}, \tilde{F}_{y m}$ are Fourier transforms of PWSF's for $E_{x}, E_{y}$ fields, respectively. Eqns (7), (8) have to be solved for unknown amplitudes of the PWSF's. PWSF (4) most distant from the slot end is a source and its magnitude equals 1 . On the slot line a respective part of these PWSF's model resulting field like a standing wave pattern. To get the complex reflection coefficient $\Gamma$ this pattern is fitted by the least squares method to the function

$$
E_{x}(y)=A\left[\exp \left(j k_{s} y\right)+\Gamma \exp \left(-j k_{s} y\right)\right]
$$

where $k_{x}$ is a propagation constant of the bound wave transmitted by the slot line [6] and $A$ is its amplitude. $E_{x}$ in (9) is a linear function of $A$ and $\Gamma$. Consequently, optimal $\Gamma$ and $A$ giving a least squares discrepancy between (9) and the calculated standing wave pattern can be found analytically. So that the reflection coefficient is

$$
\Gamma=\frac{-\sum_{i=1}^{p} \exp \left(2 j k_{s} y_{i}\right) \sum_{i=1}^{p} E_{i} \exp \left(-j k_{s} y_{i}\right)+P \sum_{i=1}^{P} E_{i} \exp \left(j k_{s} y_{i}\right)}{P \sum_{i=1}^{P} E_{i} \exp \left(-j k_{s} y_{i}\right)-\sum_{i=1}^{P} \exp \left(-2 j k_{s} y_{i}\right) \sum_{i=1}^{P} E_{i} \exp \left(j k_{s} y_{i}\right)}
$$

where $E_{i}$ are calculated values of the field along the slot sampled at points $y_{i}$, number of which is $P$.

\section{DISCUSSION OF RESULTS}

The short/open-circuited slot line is characterized most conveniently by a terminal impedance at the point where the short/open end is positioned. In adition, the impedance is normalized to the slot line characteristic impedance calculated according to the power-voltage definition. Characteristic impedance of a few slot lines discussed below is drawn in Fig. 2 . 


\subsection{Short-circuited slot line}

Calculated normalized terminal impedance of the short-circuited slot line is plotted in Fig. 3. Calculated normalized terminal resistance and reactance of the short-circuited slot line in the span of width from 0.1 to 3.25 $\mathrm{mm}$ on the substrate thick $\mathrm{h}$ with permittivity 11 and $h / \lambda_{0}$ in the range from 0.00425 to 0.0845 are given in Fig. 4. For the slot width $1.25 \mathrm{~mm}$ measured and calculated reflection losses are in Fig. 5 and agree fairly well. The difference about $1.5 \mathrm{~dB}$ between smooth calculated and rippled measured behaviour are due to an experimental configuration. This includes transitions from the waveguide to the finline, forth to the tapered slot succeeded by the measured short-circuited slot line $1.25 \mathrm{~mm}$ wide. Fig. 6 shows comparison of data obtained by the present method with those published in [1], previously in [5] and recently in [2]. A good agreement is evident. As to values shown in [3]: leaky waves are excited by the slot line $\left(\varepsilon_{\mathrm{r}}=9.8, \mathrm{~h}=1.5 \mathrm{~mm}, \mathrm{w}=0.75 \mathrm{~mm}\right)$ from app. $28 \mathrm{GHz}$ upward as follows from calculations according to [6]. Above this frequency the slot line does not transmit bound wave and consequently definition of the terminal impedance is sensless.

A model of the short-circuited slot line based on SDM is quite inconvenient for implementation into CAD packages. For such case closed-form formulae (11)-(25) come in handy. Terminal impedance $Z=R+j X$ of the short-circuited slot line normalized to the characteristic impedance of the line is

$$
\begin{aligned}
& R=A_{1} \exp \left(A_{2} u\right) \exp \left(-A_{3}\left|u-A_{4}\right|^{A_{5}}\right) \\
& X=B_{1} \exp \left(B_{2} u\right)-B_{3} \exp \left(-B_{4}\left|u-B_{5}\right|^{B_{6}}\right)
\end{aligned}
$$

where

$$
\begin{aligned}
& A_{1}=0.0727717286 q^{-0.465797325}+0.30080625 q-0.34106897 q^{2}+0.34694847 q^{3}-0.10078697 q^{4}+ \\
& +0.00988671 \mathrm{q}^{5} \\
& A_{2}=13.8297423+9.885446671 q-14.35600976 q^{2}+4.0943243 q^{3}-0.3621331 q^{4} \\
& A_{3}=0.068266118 q^{-0.97699896}+4.18954587 q-9.05401766 q^{2}+12.42629655 q^{3}-4.87911946 q^{4}+ \\
& +0.63721743 q^{5} \\
& A_{4}=0.078650476-0.00149126 q+0.0007883164 q^{2}-0.000186231 q^{3} \\
& A_{5}=-2.716486958 q^{0.93516091}+3.79099343 q-0.514345699 q^{2}+0.068801687 q^{3} \\
& \mathrm{~B}_{1}=0.319909896 \exp (0.26107124 q)+0.00391095 \exp [3.707422497(q-1.4677121)]- \\
& -0.310379366-0.0508486499 q \\
& B_{2}=737.8734162 \exp (-0.289260631 q)-697.597305+198.709714 q-22.28356943 q^{2} \\
& B_{3}=1.008786076 \exp (0.176596212 q)+0.00220244 \exp [3.423458954(q-1.11393789)]- \\
& -0.945657387-0.00104020299 \mathrm{q} \\
& B_{4}=200.4058432 \exp (-0.827944715 q)-49.27143645+64.64021387 q-16.76561181 q^{2} \\
& B_{5}=0.06252652137+0.0535727425 q-0.0513872721(q+0.021979531)^{1.0400741} \\
& B_{6}=\left(0.876001986+0.0179587974 q^{1.08985914}\right)^{-2.831023697} \\
& \mathrm{u}=\mathrm{h} / \lambda_{\mathrm{o}} \\
& q=w / h
\end{aligned}
$$

where $w$ is a width of the slot and $h$ is a thickness of the substrate. Eqns $(11)-(25)$ hold for $w \in(0.1,3.0) \mathrm{mm}$, $\mathrm{f} \in(1,18) \mathrm{GHz}, \varepsilon_{\mathrm{r}}=11$ and were tested for $\mathrm{h}=0.635$ and $1.270 \mathrm{~mm}$. 


\subsection{Open-circuited slot line}

Open-circuited slot lines terminated by two rectangular slot patches have been investigated. The former is $3.2 \times 0.6$ $\mathrm{mm}$ and the latter $4.0 \times 3.6 \mathrm{~mm}$ large. Both feeding slots are $0.15 \mathrm{~mm}$ wide. In fact, the smaller one is a slot line with an abrupt step in the slot width terminated at the end by the short. The reference plane is at point where the slot line enters into the patch. Calculated normalized terminal impedance of the open-circuited slot line terminated by these two patches is in Fig. 7. Its values recalculated into the return losses are compared with measured ones in Fig. 8. The discrepancy between calculated and measured behaviour is due to the same reason already mentioned above. If this difference is omitted both curves agree well. Resonant character of both terminations, similar to [4], is evident in Fig. 7.

\section{CONCLUSION}

A slot line terminated by the short- and open-end was analysed by the SDM. The original calculation of the reflection coefficient in [1] was modified. Reflection coefficient has been obtained faster by fitting the field distribution along the slot, described by a superposition of the PWSF's, to the standing wave pattern. Closed-form formulae of the normalized impedance of the short-circuited slot line on the substrate with permittivity 11 are presented. They result from fitting the values calculated by the SDM and facilitate much the CAD programs. Normalized terminal impedance of the open-circuited slot line with the termination in the form of the rectangular patch was calculated by the SDM. Theoretical data for both short- and open-circuited termination have been verified experimentally with good agreement.

\section{ACKNOWLEDGEMENT}

This work has been supported in part by the grants given by the Commission of European Communities under the scheme Cooperation in Science and Technology with Central and Eastern European Countries, Research Fellowships No. CIPA3510CT920643 and CIPA3510CT923158.

\section{REFERENCES}

[1] Y. Yang and N.G. Alexópoulos, "A Dynamic Model for Microstrip-Slotline Transition and Related Structures", IEEE Trans. Microwave Theory Tech., vol. MTT-36, no. 2, pp.286-293, Feb. 1988.

[2] T.Rozzi, A. Morini, A. Pallota, and F. Moglie, "A Modified Dynamic Model for Planar Microwave Circuits", IEEE Trans. Microwave Theory Tech., vol. MTT-39, no. 12, pp. 2148-2153, Dec. 1991.

[3] V.F. Fusco and Q. Chen, "Slotline Short and Open Circuit Analysis by the Finite-Diference TimeDomain Method", 24th EuMC, Cannes, France, 1994, Conference Proceedings pp. 1720-1726.

[4] J. Chramiec, "Reactances of Slotline Short and Open Circuits on Alumina Substrate", IEEE Trans. Microwave Theory Tech., vol. MTT-37, no. 10, pp. 1638-1641, Oct. 1989

[5] J. B. Knorr and J.Saenz, "End Effect in a Shorted Slot", IEEE Trans. Microwave Theory Tech., vol. MTT-21, no. 9, pp.579-580, Sept. 1973

[6] J. Macháč and J. Zehentner, "Leaky Waves on a Slot Line", URSI - International Symposium on Electromagnetic Theory, St. Petersburg, Russia, 1995, accepted for publication. 


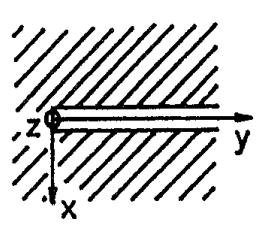

a

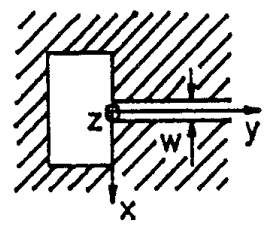

b
Fig.1 Investigated short- and open-circuited slot line

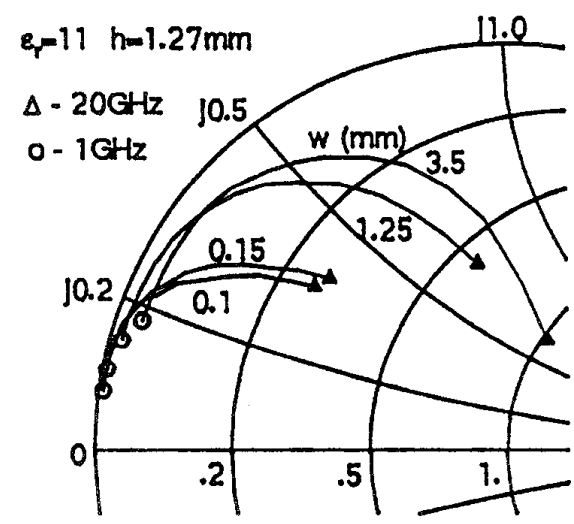

Fig.3 Normalized terminal impedance of the short-circuited slot line

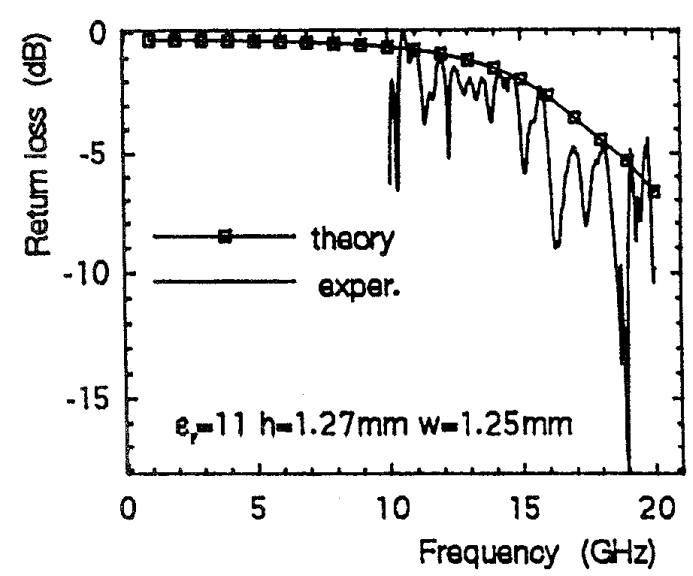

Fig.5 Reflection losses of the short-circuited slot line

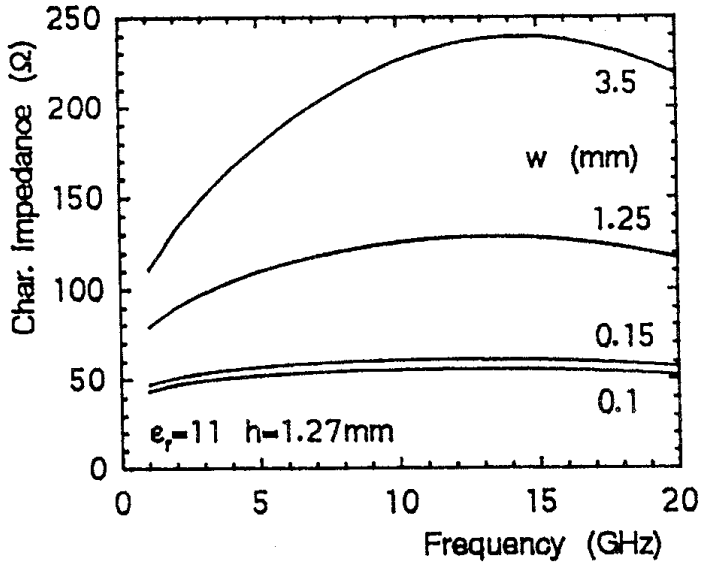

Fig.2 Characteristic impedance of the slot line

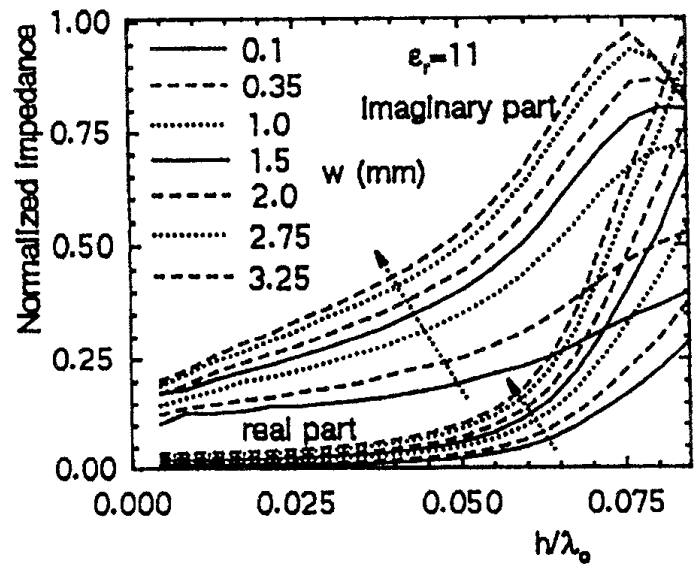

Fig.4 Normalized resistance and susceptance of the short-circuited slot line

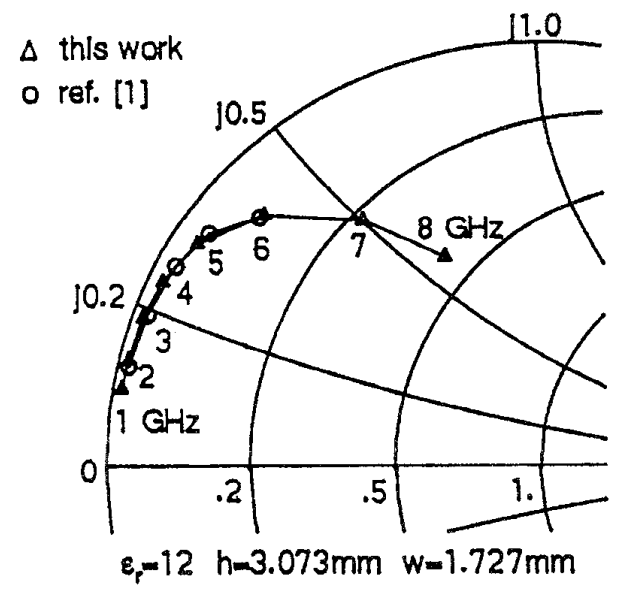

Fig.6 Comparison of normalized terminal impedance of the short-circuited slot line calculated according to the present method and that given in [1] 


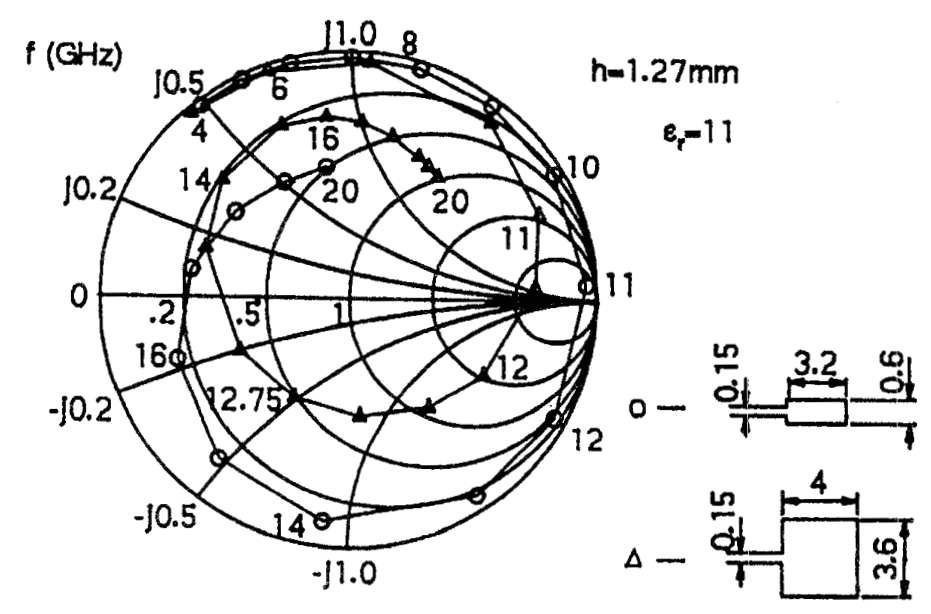

Fig. 7 Normalized terminal impedance of the open-circuited slot line

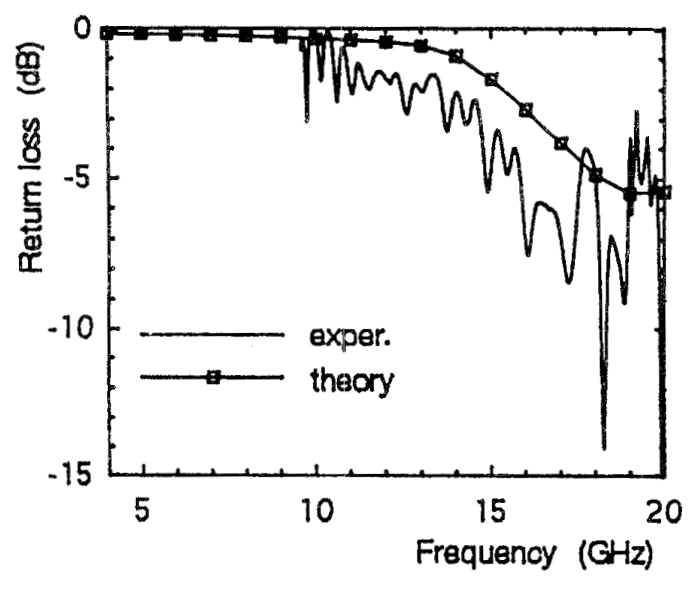

a

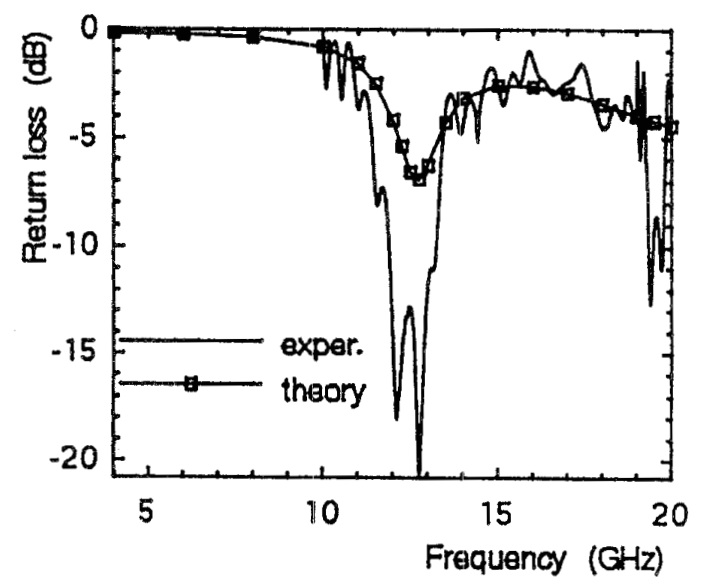

b

Fig. 8 Return losses of the open-circuited slot line a - rectangular patch $3.2 \times 0.6 \mathrm{~mm}$ b - rectangular patch $4.0 \times 3.6 \mathrm{~mm}$ 